\title{
Tensor products and Taylor's joint spectrum
}

\author{
by
}

ZOIA CEAUŞESCU* and F.-H. VASILESCU** (Bucharest)

Abstract. This work contains a refinement of a result of A. T. Dash and M. Schechter concerning the joint spectrum of tensor products of linear operators of a certain type.

1. Introduction. Let $X_{1}, \ldots, X_{n}$ be complex Banach spaces and $a$ a uniform cross norm [6] on their tensor product $X_{1} \otimes \ldots \otimes X_{n}$. Denote by $X$ the completion of $X_{1} \otimes \ldots \otimes X_{n}$ with respect to the norm $\alpha$. Suppose that $a_{j}$ is a linear continuous operator on $X_{j}$ and set $\tilde{a}_{j}=1 \otimes \ldots \otimes 1 \otimes a_{j} \otimes$ $\otimes 1 \otimes \ldots \otimes 1(j=1, \ldots, n)$. If $A$ is the bicommutant of the commuting system $\left(\tilde{a}_{1}, \ldots, \tilde{a}_{n}\right)$ in the algebra $L(X)$ of all linear bounded operators on $X$, then A. T. Dash and M. Schechter [3] have shown that the following equality holds:

$$
\sigma_{\mathcal{A}}\left(\tilde{a}_{1}, \ldots, \tilde{a}_{n}\right)=\sigma\left(a_{1}, X_{1}\right) \times \ldots \times \sigma\left(a_{n}, X_{n}\right)
$$

where $\sigma_{A}\left(\tilde{a}_{1}, \ldots, \tilde{a}_{n}\right)$ denotes the joint spectrum of $\left(\tilde{a}_{1}, \ldots, \tilde{a}_{n}\right)$ in $A$ and $\sigma\left(a_{j}, X_{j}\right)$ is the usual spectrum of $a_{j}$ as an operator on $X_{j}$.

D. Voiculescu raised within the seminar of operator theory, Institute of Mathematics, Bucharest, 1976, the following problem: Does (1.1) still hold if we take instead of $\sigma_{\mathcal{A}}\left(\tilde{a}_{1}, \ldots, \tilde{a}_{n}\right)$ the more refined joint spectrum of $\left(\dot{a}_{1}, \ldots, a_{n}\right)$ on $X$ in the sense of J. L. Taylor's [7]?

It turns out that the answer to this question is positive in the case of Hilbert spaces, and the proof of this assertion is the purpose of the present work (see also [11] for $n=2$ ).

From now on $H$ will denote a complex Hilbert space and $a=\left(a_{1}, \ldots\right.$ $\left.\ldots, a_{n}\right) \subset \mathscr{L}(H)$ a commuting system of operators on $H$. For the convenience of the reader, let us recall some definitions and results from [7], [8] and $[10]$.

Let $s=\left(s_{1}, \ldots, s_{n}\right)$ be a set of indeterminates and denote by $\Lambda[s]$ the exterior algebra, over the complex field, with the generators $s_{1}, \ldots, s_{n}$.

* National Institute for Scientifical and Technical Creation, Calea Victoriei 114, Bucharest 22.

** Theoretical Physics Laboratory, Institute of Atomic Physics, P.0. Box 5206, Bucharest.

7 - Studia Mathematỉca LXIr.3 
$\Lambda^{p}[s]$ will stand for the space of elements of degree $p$ in $\Lambda[s](p$ $=0,1, \ldots, n)$. Then we denote by $\Lambda[s, H]\left(\Lambda^{p}[s, H]\right)$ the tensor product $H \otimes \Lambda[s]\left(H \otimes \Lambda^{p}[s]\right.$, respectively). An element $x \otimes s_{v_{1}} \wedge \ldots \wedge s_{v_{p}} \in \Lambda^{p}[s, H]$ will be written simply $x s_{v_{1}} \wedge \ldots \wedge s_{v_{p}}$. The space $\Lambda^{p}[s, H]$ can be endowed with a natural structure of a Hilbert space, defining for any pair

$$
\xi=\sum_{1 \leqslant p_{1}<\ldots<v_{p} \leqslant n} x_{v_{1} \ldots v_{p}} s_{v_{1}} \wedge \ldots \wedge s_{v_{p}}
$$

and

$$
\eta=\sum_{1 \leqslant v_{1}<\ldots<v_{p} \leqslant n} y_{v_{1} \ldots v_{p}} s_{v_{1}} \wedge \ldots \wedge s_{p p}
$$

from $\Lambda^{p}[s, H]$ their scalar product

$$
\langle\zeta, \eta\rangle=\sum_{1 \leqslant v_{1}<\ldots<v_{p} \leqslant n}\left\langle x_{v_{1} \ldots v_{p}}, y_{v_{1} \ldots v_{p}}\right\rangle
$$

We have also

$$
\Lambda[s, H]=\oplus_{p=0}^{n} \Lambda^{p}[s, H] .
$$

Let us define the operator $\delta_{a}^{p}: \Lambda^{p}[s, H] \rightarrow \Lambda^{p+1}[s, H]$ by the equality

$$
\delta_{a}^{p}\left(x s_{v_{1}} \wedge \ldots \wedge s_{v_{p}}\right)=\sum_{j=1}^{n} a_{j} x s_{j} \wedge s_{v_{1}} \wedge \ldots \wedge s_{v_{p}} .
$$

Let also $\delta_{a}: A[s, H] \rightarrow \Lambda[s, H]$ be the operator $\bigoplus_{p=0}^{p} \delta_{a}^{p}$ (where $\delta_{a}^{n}=0$ : $\left.\Lambda^{n}[s, H] \rightarrow \Lambda^{0}[s, H]\right)$.

Clearly, $\delta_{a}^{p+1} \circ \delta_{a}^{p}=0$; hence $\delta_{a} \circ \delta_{a}=0$.

A basic notion in the definition of the joint spectrum of the system $a=\left(a_{1}, \ldots, a_{n}\right)$ in $H$ is that of nonsingularity [7]. Shortly, $a$ is nonsingular on $H$ iff $\operatorname{Ker} \delta_{a}=\operatorname{Im} \delta_{a}$. It is obvious that this definition makes good sense in Banach spaces [7]. However, as is pointed out in [10], there is a characterization of the nonsingularity in Hilbert spaces which makes this notion easier to be handled. Namely, the system $a$ is nonsingular on $H$ if and only if the operator $\alpha(a)=\delta_{a}+\delta_{a}^{*}$ is invertible on $A[s, H]$.

The complement of the set of all $z=\left(z_{1}, \ldots, z_{n}\right) \in C^{n}$ with the property that $z-a=\left(z_{1}-a_{1}, \ldots, z_{n}-a_{n}\right)$ is nonsingular on $H$ is called the (joint) spectrum of $a$ on $H$ [7]; this set will be denoted by $\sigma(a, H)$ in the sequel.

The spectrum $\sigma(a, H)$ of $a$ on $H$ is compact, nonempty and it is contained in the cartesian product $\sigma\left(a_{1}, H\right) \times \ldots \times \sigma\left(a_{n}, H\right)$ as well as in the joint spectrum of $\left(a_{1}, \ldots, a_{n}\right)$ in any commutative Banach algebra containing $\left(a_{1}, \ldots, a_{n}\right)$. Moreover, as in the case of Banach algebras [2], one can construct a functional calculus with analytic functions, defined in neighbourhoods of $\sigma(a, H)$; for this functional calculus a spectral mapping theorem also holds (for details see [8]).

2. The main result. If $H_{1}, \ldots, H_{n}$ are complex Hilbert spaces, we denote by $H_{1} \otimes \ldots \otimes H_{n}$ their tensor product, complete for the canonical Hilbert structure.

Let us state the main result of this paper.

2.1. THeorem. Assume that $H_{1}, \ldots, H_{n}$ are complex Hilbert spaces, $H=H_{1} \otimes \ldots \otimes H_{n}, a_{j} \in \mathscr{L}\left(H_{j}\right), \quad \tilde{a}_{j}=1 \otimes \ldots \otimes 1 \otimes a_{j} \otimes 1 \otimes \ldots \otimes 1 \quad$ $=1, \ldots, n)$ and $\tilde{a}=\left(\tilde{a}_{1}, \ldots, \tilde{a}_{n}\right) \subset \mathscr{L}(H)$. Then we have the equality

$$
\sigma(\tilde{a}, H)=\sigma\left(a_{1}, H_{1}\right) \times \ldots \times \sigma\left(a_{n}, H_{n}\right) .
$$

In order to prove Theorem 2.1 we need an auxiliary result.

2.2. Lemina. Let $H$ and $K$ be Hilbert spaces, $a=\left(a_{1}, \ldots, a_{n}\right) \subset \mathscr{L}(H)$ a commuting system, $a_{j}^{\prime}=a_{j} \otimes 1 \subset \mathscr{L}(H \otimes K) \quad(j=1, \ldots, n)$ and $a^{\prime}$ $=\left(a_{1}^{\prime}, \ldots a_{n}^{\prime}\right)$. Then the operators $\alpha\left(a^{\prime}\right) \in \mathscr{L}(\Lambda[s, H \otimes K])$ and $\alpha(a) \otimes 1$ $\in \mathscr{L}(\Lambda[s, H] \otimes K)$ are unitarily equivalent.

Proof. Let us remark that we can define a unitary map

by the relation

$$
u_{p}: \Lambda^{p}[s, H \otimes K] \rightarrow \Lambda^{p}[s, H] \otimes K
$$

$$
u_{p}\left(x \otimes y s_{v_{1}} \wedge \ldots \wedge s_{v_{p}}\right)=\left(x s_{v_{1}} \wedge \ldots \wedge s_{v_{p}}\right) \otimes y .
$$

Then $u=\bigoplus_{p=0}^{n} u_{p}: \Lambda[s, H \otimes K] \rightarrow \Lambda[s, H] \otimes K$ is unitary. Moreover, we have

$$
\begin{aligned}
& u_{p+1} \delta_{a^{\prime}}^{p}\left(x \otimes y s_{v_{1}} \wedge \ldots \wedge s_{v_{p}}\right)=u_{p+1}\left(\sum_{j=1}^{n} a_{j} x \otimes y s_{j} \wedge s_{v_{1}} \wedge \ldots \wedge s_{v_{p}}\right) \\
& =\left(\sum_{j=1}^{n} a_{j} x s_{j} \wedge s_{v_{1}} \wedge \ldots \wedge s_{v_{p}}\right) \otimes y=\left(\delta_{a}^{p} \otimes 1\right) u_{p}\left(x \otimes y s_{v_{1}} \wedge \ldots \wedge s_{v_{p}}\right) ;
\end{aligned}
$$

hence $u \delta_{a^{\prime}}=\left(\delta_{a} \otimes 1\right) u$. From this we obtain

$$
\alpha(a) \otimes 1=\delta_{a} \otimes 1+\delta_{a}^{*} \otimes 1=u\left(\delta_{a^{\prime}}+\delta_{a^{\prime}}^{*}\right) u^{*}=u \alpha\left(a^{\prime}\right) u^{*} ;
$$

hence $\alpha(a) \otimes 1$ and $\alpha\left(a^{\prime}\right)$ are unitarily equivalent.

2.3. Corollary. With the conditions of Lemma 2.2, if $K \neq 0$, we have the equality

$$
\sigma\left(a^{\prime}, H \otimes K\right)=\sigma(a, H) .
$$

Proof. Indeod, by Lemma 2.2, $\alpha\left(a^{\prime}\right)$ is invertible if and only if $\alpha(a) \otimes 1$ is invertible, hence if and only if $\alpha(a)$ is invertible (see, for example, [5], Lemma 4.1).

Proof of Theorem 2.1. We may write

$\sigma(\tilde{a}, H) \subset \sigma\left(\tilde{a}_{1}, H\right) \times \ldots \times \sigma\left(\tilde{a}_{n}, H\right)=\sigma\left(\boldsymbol{a}_{1}, H_{1}\right) \times \ldots \times \sigma\left(a_{n}, H_{n}\right)$, 
the last equality being true by virtue of [5], Lemma 4.1; therefore we have one inclusion in (2.1).

The reverse inclusion will be proved by induction with respect to $n$. For $n=1$ the relation (2.1) is trivial. Assume that the property is valid for $n$ and let us get it for $n+1$. Let $H_{n+1}$ be an arbitrary Hilbert space and take $a_{n+1} \in \mathscr{L}\left(H_{n+1}\right)$. Denote by $H^{\prime}$ the space $H_{1} \otimes \ldots \otimes H_{n} \otimes H_{n+1}$ $=H \otimes H_{n+1}$ and by $s^{\prime}$ the system of indeterminates $\left(s_{1}, \ldots, s_{n}, s_{n+1}\right)$ $=s \cup\left(s_{n+1}\right)$. Consider the systems of operators $\tilde{a}^{\prime}=\left(\tilde{a}_{1}^{\prime}, \ldots, \tilde{a}_{n}^{\prime}\right)$ and $\tilde{b}=\left(\tilde{a}_{1}^{\prime}, \ldots, \tilde{a}_{n}^{\prime}, \tilde{a}_{n+1}^{\prime}\right)$, with $\tilde{a}_{j}^{\prime} \in \mathscr{L}\left(H^{\prime}\right)$, where $\tilde{a}_{j}^{\prime}=1 \otimes \ldots \otimes 1 \otimes a_{j} \otimes 1 \otimes$ $\otimes \ldots \otimes 1(j=1, \ldots, n+1)$.

Notice that we have the decomposition

$$
\Lambda\left[s^{\prime}, H^{\prime}\right]=\Lambda\left([s, H] \otimes \tilde{s}_{n+1} \Lambda\left[s, H^{\prime}\right],\right.
$$

where $\tilde{s}_{n+1} \in \mathscr{L}\left(\Lambda\left[s^{\prime}, H^{\prime}\right]\right)$ is given by

$$
\text { - } \quad \tilde{s}_{n+1}(\xi)=s_{n+1} \wedge \xi, \quad \xi \in \Lambda\left[s^{\prime}, H^{\prime}\right] \text {. }
$$

Then the matrix of $\delta_{\tilde{b}}$ with respect to the decomposition (2.2) has the form

therefore

$$
\delta_{\tilde{b}}=\left[\begin{array}{ll}
\delta_{\tilde{a}^{\prime}} & 0 \\
\tilde{a}_{n+1} \tilde{s}_{n+1} & \delta_{\tilde{a}^{\prime}}
\end{array}\right] ;
$$

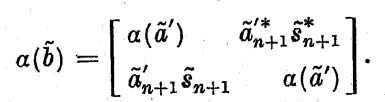

According to the proof of Lemma 2.2, the space $\Lambda\left[s, H^{\prime}\right]$ can be identified with $\Lambda[s, H] \otimes H_{n+1}$ and the operator $\alpha\left(\tilde{a}^{\prime}\right)$ with $\alpha(\tilde{a}) \otimes 1$.

Analogously, the operator $\tilde{a}_{n+1}^{t} \tilde{s}_{n+1}$ can be identified with $\tilde{s}_{n+1} \otimes a_{n+1}$, therefore the matrix (2.3) is unitarily equivalent to the matrix

$$
\left[\begin{array}{ll}
\alpha(\tilde{a}) \otimes 1 & \tilde{s}_{n+1}^{*} \otimes a_{n+1}^{*} \\
\tilde{s}_{n+1} \otimes a_{n+1} & \alpha(\tilde{a}) \otimes 1
\end{array}\right],
$$

defined on the space $\left(\Lambda[s, H] \otimes H_{n+1}\right) \otimes\left(\tilde{s}_{n+1} \Lambda[s, H] \otimes H_{n+1}\right)$. In this way, if $\tilde{b}$ is nonsingular on $H^{\prime}$, then both (2.4) and

$$
\left[\begin{array}{ll}
\alpha(\tilde{a}) \otimes 1 & \tilde{s}_{n+1}^{*} \otimes a_{n+1} \\
\tilde{s}_{n+1} \otimes a_{n+1}^{*} & \alpha(\tilde{a}) \otimes 1
\end{array}\right]
$$

are invertible, where (2.5) is (2.4) written for $\tilde{b}^{*}$, which is also nonsingular. In order to prove the inclusion

$$
\sigma\left(a_{1}, H_{1}\right) \times \therefore \times \sigma\left(a_{n}, H_{n}\right) \times \sigma\left(a_{n+1}, H_{n+1}\right) \subset \sigma\left(\tilde{b}, H^{\prime}\right),
$$

it will be enough to show that if

$$
(0, \ldots, 0) \in \sigma\left(a_{1}, H_{1}\right) \times \ldots \times \sigma\left(a_{n}, H_{n}\right) \times \sigma\left(a_{n+1}, H_{n+1}\right),
$$

then $(0, \ldots, 0) \in \xi\left(\tilde{b}, H^{\prime}\right)$. Since we have, in particular, $(0, \ldots, 0)$ $\in \sigma\left(a_{1}, H_{1}\right) \times \ldots \times \sigma\left(a_{n}, H_{n}\right)$, we obtain, by the induction hypothesis, $(0, \ldots, 0) \in \sigma(\tilde{a}, H)$. At the same time, we have also $0 \in \sigma\left(a_{n+1}, H_{n+1}\right)$.

Suppose, however, that $\tilde{b}$ is nonsingular; hence $a(\tilde{b})$ is invertible, and let us show that this assumption leads to a contradiction. We have to discuss some cases.

(i) If $\overline{\alpha(\tilde{a}) \Lambda[s, H]}=\Lambda[s, H]$ and $\overline{a_{n+1} H_{n+1}}=H_{n+1}$, then there are two sequences $\left\{\xi_{k}\right\}_{l_{c}} \subset \Lambda[s, H],\left\|\xi_{k_{k}}\right\|=1$ and $\left\{\eta_{k}\right\}_{k} \subset H_{n+1},\left\|\eta_{k}\right\|=1$, stich that $\alpha(\tilde{a}) \xi_{k} \rightarrow 0$ and $a_{n+1} \eta_{k} \rightarrow 0$ as $k \rightarrow \infty$. Then the sequence

$$
\left(\xi_{k} \otimes \eta_{k}\right) \oplus 0 \in\left(\Lambda[s, H] \otimes H_{n+1}\right) \oplus\left(\tilde{s}_{n+1} \dot{A}[s, H] \otimes H_{n+1}\right)
$$

has the properties

and

$$
\left\|\left(\xi_{k} \otimes \eta_{k}\right) \oplus 0\right\|=\left\|\xi_{k} \otimes \eta_{k}\right\|=\left\|\xi_{k}\right\|\left\|\eta_{k}\right\|=1
$$

$$
\left[\begin{array}{ll}
\alpha(\tilde{a}) \otimes 1 & \tilde{s}_{n+1}^{*} \otimes a_{n+1}^{*} \\
\tilde{s}_{n+1} \otimes a_{n+1} & \alpha(\tilde{a}) \otimes 1
\end{array}\right]\left[\begin{array}{c}
\xi_{k} \otimes \eta_{k} \\
0
\end{array}\right] \rightarrow 0 \quad(k \rightarrow \infty),
$$

and this is not possible because (2.4) is invertible.

(ii) If $\overline{\alpha(\tilde{a}) \Lambda[s, H]}=\Lambda[s, H]$ and $\operatorname{Ker} a_{n+1}^{*} \neq 0$, then there is a sequence $\left\{\xi_{l i}\right\}_{l_{0}} \subset A[s, H]$ as above and a vector $\eta \in H_{n+1},\|\eta\|=1$, such that $a_{n+1}^{*} \eta=0$. Then the sequence

$$
\left(\xi_{k} \otimes \eta\right) \oplus 0 \in\left(\Lambda[s, H] \otimes H_{n+1}\right)+\left(\tilde{s}_{n+1} \Lambda[s, H] \otimes H_{n+1}\right)
$$

has the properties $\left\|\left(\xi_{k} \otimes \eta\right) \oplus 0\right\|=1$ and

$$
\left[\begin{array}{ll}
\alpha(\tilde{a}) \otimes 1 & \tilde{s}_{n+1}^{*-} \otimes a_{n+1} \\
\tilde{s}_{n+1} \otimes a_{n+1}^{*} & \alpha(\tilde{a}) \otimes 1
\end{array}\right]\left[\begin{array}{c}
\xi_{k} \otimes \eta \\
0
\end{array}\right] \rightarrow 0 \quad(k \rightarrow \infty),
$$

which is a contradiction since (2.5) is invertible.

(iii) If $\operatorname{Ker} \alpha(\tilde{a})^{*}=\operatorname{Ker} \alpha(\tilde{a}) \neq 0$ and $\overline{a_{n+1} H_{n+1}}=H_{n+1}$, then there is a vector $\xi \in \Lambda[s, H],\|\xi\|=1$, such that $\alpha(\tilde{a}) \xi=0$ and a sequence $\left\{\eta_{k}\right\}_{k} \subset H_{n+x}$ as in casse (i). A similar argument to that used in case (i) shows that this situation is again impossible.

(iv) If $\operatorname{Kor} \alpha(\tilde{a}) \neq 0$ and $\operatorname{Ker} a_{n+1}^{*} \neq 0$, then $\alpha(\tilde{a}) \xi=0$ and $a_{n+1}^{*} \eta=0$ for some $\xi \in \Lambda[s, H]$ and $\eta \in H_{n+1}$ with $\|\xi\|=\|\eta\|=1$, and then the vector $(\xi \otimes \eta) \oplus 0 \neq 0$ is an eigenvalue of the matrix (2.5), which contradicts the nonsingularity of $\tilde{b}$.

In this manner we have proved that the non-invertibility of both $a(\tilde{a})$ and $a_{n+1}$ implies the non-invertibility of $a(\tilde{b})$, and this finishes the proof of our theorem. 
2.4. Corollary. With the conditions of Theorem 2.1, if $f(z)$ is any analytic function in an open neighbourhood $G$ of $\sigma(\tilde{a}, H)$ in $C^{n}$, then there is a system of open sets $\left(G_{1}, \ldots, G_{n}\right)$ in $C$ such that

$$
\sigma(\tilde{a}, H) \subset G_{1} \times \ldots \times G_{n} \subset G,
$$

and a system of finite families of Jordan curves $\left(\Gamma_{1}, \ldots, \Gamma_{n}\right), \Gamma_{j}$ surrounding $\sigma\left(a_{j}, H_{j}\right), \Gamma_{j} \subset G_{j}(j=1, \ldots, n)$ with the property

(2.6) $f\left(\tilde{a}_{1}, \ldots, \tilde{a}_{n}\right)=$

$\frac{1}{(2 \pi i)^{n}} \int_{\Gamma_{1}} \ldots \int_{\Gamma_{n}} f\left(z_{1}, \ldots, z_{n}\right)\left(z_{1}-a_{1}\right)^{-1} \otimes\left(z_{2}-a_{2}\right)^{-1} \otimes \ldots \otimes\left(z_{n}-a_{n}\right)^{-1} d z_{1} \ldots d z_{n}$,

where the left term of (2.6) is calculated by any functional caloulus with analytic functions [8].

Proof. The existence of the sets $G_{1}, \ldots, G_{n}$ and of the curves $\Gamma_{1}, \ldots, \Gamma_{n}$ follows from equality (2.1).

It is clear that the right term of (2.6) defines a functional calculus with analytic functions in the open set $G_{1} \times \ldots \times G_{n} \supset \sigma(a, H)$. Since $G_{1} \times \ldots \times G_{n}$ is a holomorphy domain, it follows from a result of J. L. Taylor [9], Prop. 4.8, that the functional calculus is uniquely determined, hence (2.6) holds (see also [4] for some connections).

2.5. Corollary. With the conditions of Corollary 2.4 we have the formula

$$
\sigma\left(f\left(\tilde{a}_{1}, \ldots, \tilde{a}_{n}\right)\right)=f\left(\sigma\left(a_{1}, H_{1}\right), \ldots, \xi\left(a_{n}, H_{n}\right)\right) .
$$

Proof. Fornula (2.7) follows from the spectral mapping theorem ([8], Th. 4.8).

2.6. CoRollaRY. If $a_{j} \in \mathscr{L}\left(H_{j}\right)$ are arbitrary $(j=1, \ldots, n)$ and $H=H_{1} \otimes \ldots \otimes H_{n}$, then

(2.8) $\quad \sigma\left(a_{1} \otimes \ldots \otimes a_{n}, H\right)=\sigma\left(a_{1}, H_{1}\right) \times \ldots \times \sigma\left(a_{n}, H_{n}\right) \quad$ ([1]).

Proof. Formula (2.8) is a particular case of (2.7) with $f\left(z_{1}, \ldots, z_{n}\right)$ $=z_{1} \ldots z_{n}$, by noticing that $a_{1} \otimes \ldots \otimes a_{n}=\tilde{a}_{1} \ldots \tilde{a}_{n}$.

\section{References}

[1] A. Brown and C. Pearcy, Spectra of tensor product of operators, Proc. Amer. Math. Soc. 17 (1966), pp. 162-166.

[2] N. Bourbaki, Théories spectrales, Hermann, Paris 1967.

[3] A. T. D ash and M. Schech ter, Tensor products and joint spectra, Israel J. Math. 8 (1970), pp. 191-193.

[4] M. R. E mbry and M. Rosenblum, Spectra, tensor products, and linear operator equations, Pacific J. Math. 53 (1974), pp. 95-107.
[5] T. Ichinose, On the spectra of tensor products of linear operalors in Banach spaces, J. Reine Angew. Math. 244 (1970), pp. 119-153.

[6] R. Schatten, A theory of cross-spaces, Princeton 1950

[7] J. L. Taylor, A joint spectrum for several commuting operators, J. Functional Anal. 6 (1970), pp. 172-191.

[8] - The analytic functional calculus for several commuting operators, Acta Math. 125 (1970), pp. 1-38.

[9] - A general framework for a multi-operator functional calculus, Advances in Math. 9 (1972), pp. 183-252.

[10] F.-H. Vasilescu, A characterization of the joint spectrum in Hilbert spaces, Rev. Roum. Math. Pures Appl. 22 (1977), pp. 1003-1009,

[11] - On pairs of commuting operators, Studia Math. 62 (1977), pp. 201-205. 